\title{
Magnitude Squared of Coherence to Detect Imaginary Movement
}

\author{
Sady Antônio Santos Filho, ${ }^{1}$ Carlos Julio Tierra-Criollo, ${ }^{1}$ Ana Paula Souza, ${ }^{1}$ \\ Marcos Antonio Silva Pinto, ${ }^{1}$ Maria Luiza Cunha Lima, ${ }^{2}$ and Gilberto Mastrocola Manzano ${ }^{3}$ \\ ${ }^{1}$ Biomedical Engineering Studies and Research Group (NEPEB), Department of Electrical Engineering, \\ Federal University of Minas Gerais (UFMG), Av. Antônio Carlos 6627, 31270-010 Belo Horizonte, MG, Brazil \\ ${ }^{2}$ Department of Arts and Social Sciences, Federal University of Minas Gerais (UFMG), Av. Antônio Carlos 6627, \\ 31270-010 Belo Horizonte, MG, Brazil \\ ${ }^{3}$ Department of Neurology, Federal University of São Paulo (UNIFESP), Rua Botucatu 603, 04023-062 São Paulo, SP, Brazil
}

Correspondence should be addressed to Sady Antônio Santos Filho, sasfilho@planetarium.com.br

Received 1 January 2009; Revised 3 April 2009; Accepted 8 June 2009

Recommended by Karim Oweiss

\begin{abstract}
This work investigates the Magnitude Squared of Coherence (MSC) for detection of Event Related Potentials (ERPs) related to left-hand index finger movement. Initially, ERP presence was examined in different brain areas. To accomplish that, 20 EEG channels were used, positioned according to the 10-20 international system. The grand average, resulting from 10 normal subjects showed, as expected, responses at frontal, central, and parietal areas, particularly evident at the central area $(\mathrm{C} 3, \mathrm{C} 4, \mathrm{Cz})$. The MSC, applied to movement imagination related EEG signals, detected a consistent response in frequencies around $0.3-1 \mathrm{~Hz}$ (delta band), mainly at central area $(\mathrm{C} 3, \mathrm{Cz}$, and $\mathrm{C} 4)$. Ability differences in control imagination among subjects produced different detection performance. Some subjects needed up to 45 events for a detectable response, while for some others only 10 events proved sufficient. Some subjects also required two or three experimental sessions in order to achieve detectable responses. For one subject, response detection was not possible at all. However, due to brain plasticity, it is plausible to expect that training sessions (to practice movement imagination) improve signal-noise ratio and lead to better detection using MSC. Results are sufficiently encouraging as to suggest further exploration of MSC for future BCI application.
\end{abstract}

Copyright (C) 2009 Sady Antônio Santos Filho et al. This is an open access article distributed under the Creative Commons Attribution License, which permits unrestricted use, distribution, and reproduction in any medium, provided the original work is properly cited.

\section{Introduction}

Millions of people suffer from severe impairments due to physical, sensorial, or mental damage [1]. When neuromuscular channels that permit communication between brain and environment are affected, partial or total muscle control may be lost. A typical disease affecting these channels is amyotrophic lateral sclerosis (ALS), characterized by motor neurons degeneration and the consequent loss of voluntary muscles control $[2,3]$.

A considerable amount of research has been devoted to improving the quality of life of patients suffering from such afflictions. The investigations have the purpose of providing them with access to regular services and opportunities, especially by means of the development and improvement of systems that create new means of communication between patients and their external environment.
Among these projects, Brain-Computer Interface (BCI) has called attention to itself by its promising results. This interface provides a direct pathway for control of the patient's external world without reliance on muscle control.

BCI can be defined as a technical system through which a person or animal sends information from the brain to a computer (microprocessor systems) that processes this information and, thus, uses it for controlling an external control device. The information, generated by brain activity, can be registered by different techniques, such as electroencephalography, magnetoencephalography, positron emission tomography, functional magnetic resonance imaging, and electrocorticography. Due to the relative low cost and ease of usability, electroencephalography and electrocorticography are some of the most widely used techniques. However, since electrocorticography is invasive, there are some inherent risks related to the surgical procedures required. BCIs utilizing 


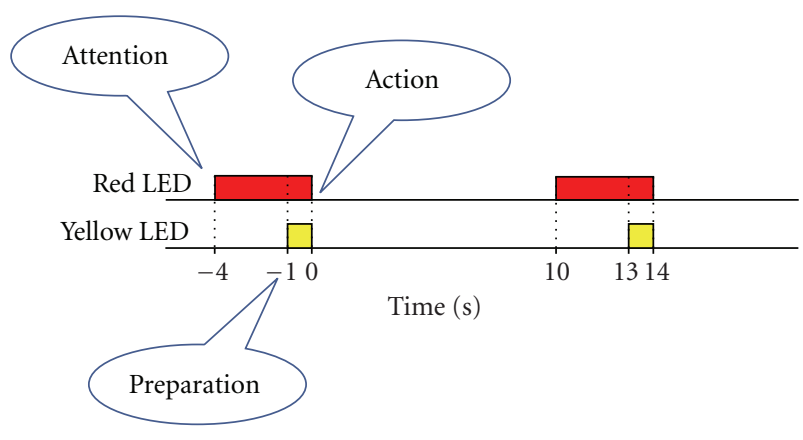

FIgURE 1: Event protocol. The red LED suggests attention, and the yellow LED suggests preparation for movement or preparation for movement imagination. Subjects are instructed to initiate the task when both LEDs are turned off ( $t=0$ second).

this technique have been mostly confined to animals [46]. Nonetheless, there have already been some experiments using electrocorticography on humans [7-11].

With regards to electroencephalography, the electric potential, generated by large neuron groups, with dendrite trees perpendicular to the skull is registered in different scalp areas [12]. The signals obtained, using this method, are referred to as an electroencephalogram (EEG). This type of signal has high temporal resolution but poor spatial localization due to the spread of electrical brain activity, because of the volume conductor between the brain sources and scalp. Furthermore, the number of recording electrodes is limited. In electrocorticography, in which outcoming signals are referred to as an electrocorticogram (ECoG), microelectrodes are implanted inside the cortex to record the action potential of an individual neuron or relatively small neuron groups. Recording the signal directly from cortex makes it less susceptible to artifacts and displays high temporal and spatial resolution.

The goal of BCI techniques is to obtain the eventrelated potential (ERP)-for instance, that related to visual stimulation, movement, and movement imagination - and use it to control an external device. The most important challenge, therefore, is the identification of the ERP amidst the "spontaneous" brain activity; whereas the ERP amplitude is usually of some microvolts, spontaneous activity can reach hundreds of microvolts.

Various techniques have been proposed to detect ERPs in spontaneous EEG, both in time and frequency domain. Among them are Fast-Fourier Transform [13], Wavelet Transform [10, 14-16], parametric modeling [12, 17-19], neural networks $[4,12,20]$, event-synchronized epochs (EEG segments synchronized with events) averaging [7, 21], event-related desynchronization and synchronization [22$24]$, linear discriminant analysis [14], principal components and independent components [25-28], and other kinds of filters, such as spatial filters $[13,14,29]$.

Although some of these techniques present good outcomes with invasive BCIs (ECoG), noninvasive (EEG) application still requires elevated processing times and a great number of EEG epochs $[18,30]$. In order to improve
ERP detection, different techniques have been enhanced and combined $[11,14]$.

The statistical detection technique magnitude-squared coherence (MSC) has been applied to the identification of event-related synchronization responses (time and phase). MSC has been proven very effective in the detection of somatosensory [31], auditory [32], and visual evoked potentials $[33,34]$, reducing the number of epochs necessary for response detection. A reason for this is detector's robustness, since its firing threshold is relatively independent of response shape [35]. This work investigates the MSC use for ERP detection, both due to left-hand index finger movement and movement imagination. Our main goal is to determine those ERPs frequency bands as well as to identify brain areas where this detection is more consistent and useful for future BCI applications.

\section{Materials and Methods}

Ten neurologically healthy volunteers (6 males, $21-46$ years) participated in the study. Subjects were not under the influence of any medication that could interfere with EEG. During data recording, subjects remained comfortably seated in an armchair, with head, shoulders, and arms resting on the backrest. Light was adjusted to avoid signal interference, and room temperature was kept at $27 \pm 1.7^{\circ} \mathrm{C}$. The experiment was run at Biomedical Engineering Laboratory and approved by the local Ethics Committee. All participants read and signed an informed consent form.

The EEG was recorded from 20 scalp electrodes $(\mathrm{Ag} / \mathrm{AgCl}$ and diameter of $10 \mathrm{~mm}$ ), placed according to International 10-20 System (Fp1, Fp2, F7, F3, Fz, F4, F8, T3, C3, Cz, C4, T4, T5, P3, Pz, P4, T6, O1, Oz, and O2) with reference at earlobes (A1 and A2 electrodes). Signals were recorded using a 36-channel BrainNet BNT-36 (EMSA) biological amplifier, band-pass filtered $(0.1-100 \mathrm{~Hz})$ and digitized at $600 \mathrm{~Hz}$.

During EEG recording, two Light Emitter Diodes (LEDs), one yellow and one red, were used to prompt the events related to movement or movement imagination as shown in Figure 1. Both LEDs were displayed on a rectangular $(30 \times 20 \mathrm{~cm})$ black panel, placed in the subjects eye line angle of vision, at $80 \mathrm{~cm}$ distance.

EEG recording was made during four separate sessions with all subjects: (1) spontaneous activity with no LED light, (2) spontaneous activity with LEDs turned on, (3) movement of the left index finger, and (4) same finger movement imagination. Each session was conducted once with the subject with the exception of the imagination session, which was conducted twice. For two subjects it was necessary to conduct an additional imagination session.

The movement and movement imagination sessions lasted for 20 minutes, while spontaneous EEG sessions were 15 minutes long. Recording was performed on two nonconsecutive days for each subject. During the first day, the sessions of movement and spontaneous EEG with LEDs turned on were realized, and on the second day, movement imagination and spontaneous EEG with LEDs turn off were realized. 
For movement-EEG synchronization, an integrated Microelectro Mechanical System (iMEMS) accelerometer, (Analog Devices-ADXL 213), with full-scale range of $\pm 1.2 \mathrm{~g}$, was positioned on distal phalanx of the left index finger. Signals from accelerometer and the yellow LED triggers were recorded by the BrainNET-36. The data were processed off-line, with the software MATLAB (MathWorks).

For the movement session, the subject was asked to move his (her) left index finger up and down. For the movement imagination session, the procedure was the same, but subjects were to only imagine the movement instead of actually performing it. Each subject made 85 repetitions of movement or movement imagination in the course of each 20 minute session.

Throughout spontaneous EEG recording, subjects were oriented to look at the LEDs, avoiding movement and movement imagination.

2.1. Data Preprocessing. Signals were filtered through a Butterworth digital filter of the 2nd order, high-pass cutoff frequency at $0.1 \mathrm{~Hz}$ and low-pass cutoff frequency at $100 \mathrm{~Hz}$. After filtering, mean value of each epoch (EEG segment between -0.7 second and 2 seconds-Figure 1 ) was removed. After that the algorithm for automatic rejection of epochs showing strong artifacts was applied [31]. For this, a 15-second EEG segment (artifact free) was selected as reference for signal levels and for signal standard deviation $(\sigma)$ estimation. A threshold value of $3 \sigma$ was then defined and applied to the epochs of the EEG signal. Epochs in which a continuous segment of more than $5 \%$ of the samples, or a total of $10 \%$ of samples exceeded the threshold, were rejected as containing artifact. With the recording of spontaneous EEG for unlighted and light LEDs (15 minutes) 60 epochs were obtained, and with the recording of movement and imagination sessions (20 minutes) 85 epochs were obtained. After artifact rejection, the number of epochs was greater than 45 , except with frontal channels (mean of 20 epochs). Thus, in this work 45 epochs were randomly selected.

2.2. Data Processing. In order to identify brain areas that presented Event Related Potentials (ERPs) due to left-hand index finger movement and movement imagination, an eventsynchronized epochs averaging technique $[7,21]$ was applied to each EEG signal from each different channel. The grand average (using artifact free segments), for each channel, was determined for four different situations: spontaneous EEG with lighted LEDs, spontaneous EEG without lighted LEDs, actual movement, and movement imagination.

Objective response detection was obtained through magnitude-squared coherence (MSC). This method is based on a coherence function between two signals $y(n)$ and $x(n)$ [31-36] that is defined by

$$
\gamma_{y x}(f)=\frac{P_{y x}(f)}{\sqrt{P_{y y}(f)} \sqrt{P_{x x}(f)}},
$$

where $P_{y x}(f)$ is the cross power spectral density (PSD), and $P_{y y}(f)$ and $P_{x x}(f)$ are their individual PSDs. The coherence function can be perceived as the spectrum of cross correlation between signals and indicates the linear dependency between theirs harmonics. Taking the square of absolute value of (1) we obtain

$$
\kappa(f)=\left|\gamma_{y x}(f)\right|^{2}=\frac{\left|P_{y x}(f)\right|^{2}}{P_{y y}(f) P_{x x}(f)} .
$$

This value is a real number. The $\kappa(f)$ function estimation for ergodic signals can be the following [31-36]:

$$
\widehat{\kappa}(f)=\frac{\left|\sum_{i=1}^{M} Y_{i}(f) X^{*}{ }_{i}(f)\right|^{2}}{\sum_{i=1}^{M}\left|Y_{i}(f)\right|^{2} \sum_{i=1}^{M}\left|X_{i}(f)\right|^{2}},
$$

where $*$ indicates the complex conjugate, $Y_{i}(f)$ and $X_{i}(f)$ are the $T$-length Fourier Transform of the $i$ th windowed epochs, and $M$ is the number of epochs used in the estimation.

Taking $y(n)$ as identical events in all epochs (e.g., sensorial stimulus, tasks) and $x(n)$ the EEG signal from the scalp, the estimation of $\kappa(f)$ can be simplified and denoted as the magnitude square coherence, $\operatorname{MSC}(f)$ [31-36]:

$$
\operatorname{MSC}(f)=\frac{\left|\sum_{i=1}^{M} X_{i}(f)\right|^{2}}{M \sum_{i=1}^{M}\left|X_{i}(f)\right|^{2}}
$$

In the absence of a consistent response to the event (only spontaneous EEG), the numerator is low, and the MSC approaches 0 , while $M$ tends toward infinity. On the other hand, when a response is present, MSC tends to 1 while $M$ tends to infinity.

For $M$ independent epochs of a random Gaussian signal (i.e., no event response), the MSC can be considered to be related to the $F$-distribution by [31-36]

$$
\operatorname{MSC}(f) \sim \frac{F_{2,2 M-2}}{M-1+F_{2,2 M-2}},
$$

where we can obtain the critical value for a significance level of $\alpha$, for the null-hypothesis of no-response by

$$
\mathrm{MSC}_{\mathrm{crit}}=\frac{F_{2,2 M-2 \alpha}}{M-1+F_{2,2 M-2 \alpha}} .
$$

For the desired significance level, the critical value constitutes threshold bellow which coherence can be assumed not to be significantly different to zero. According to [36], this critical value is related to $M$ by

$$
\mathrm{MSC}_{\text {crit }}=1-\alpha^{1 /(M-1)} \text {. }
$$

In the presence of a event-related synchronization response, in the $f$ frequency, a positive $\operatorname{detection}(\operatorname{MSC}(f)>$ $\left.\mathrm{MSC}_{\text {crit }}\right)$ is expected. In the absence of a response or in the no-event condition, a false positive rate of $\alpha$ is expected in all frequencies. Equation (5) is not valid for the DC component and the Nyquist frequency. 


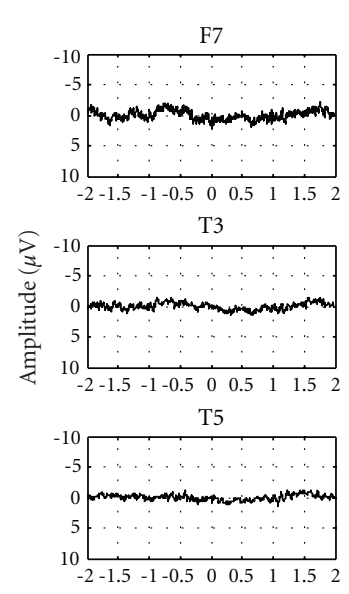

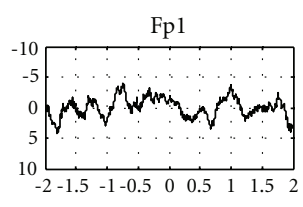

F3

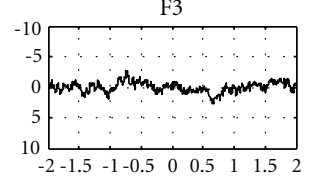

C3

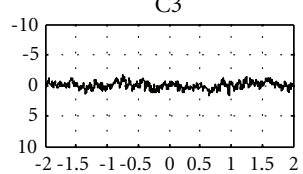

P3

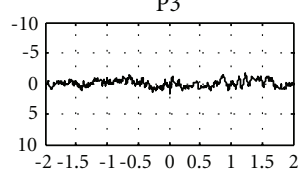

$\mathrm{O} 1$

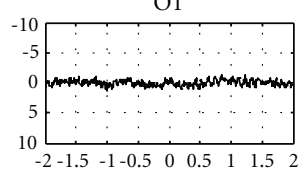

$\mathrm{Fz}$

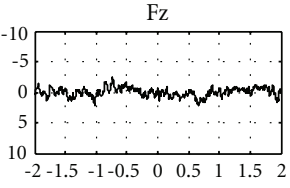

$\mathrm{Cz}$

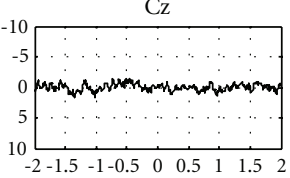

$\mathrm{Pz}$

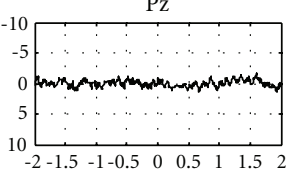

$\mathrm{Oz}$

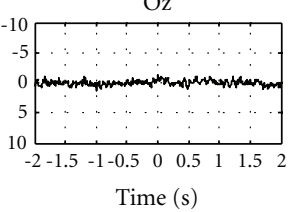

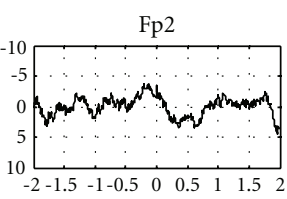

F4

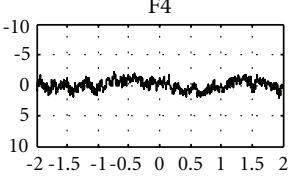

C4

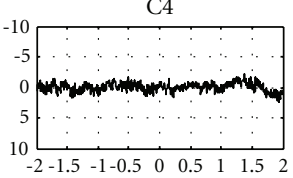

$\mathrm{P} 4$

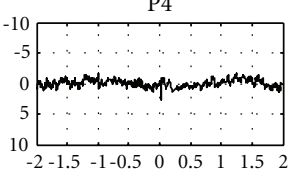

$\mathrm{O} 2$

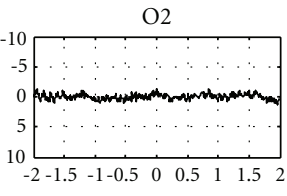

F8

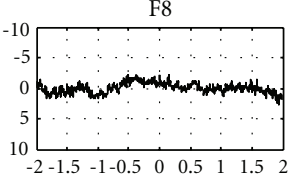

$\mathrm{T} 4$

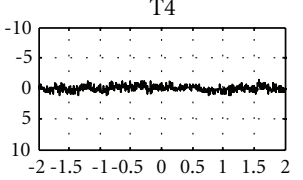

T6

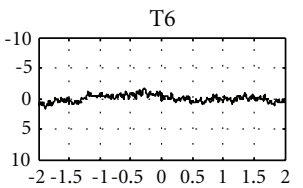

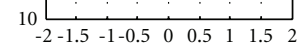

FIGURE 2: Grand Average topographic distribution of ERPs from spontaneous EEG, with unlighted LEDs.
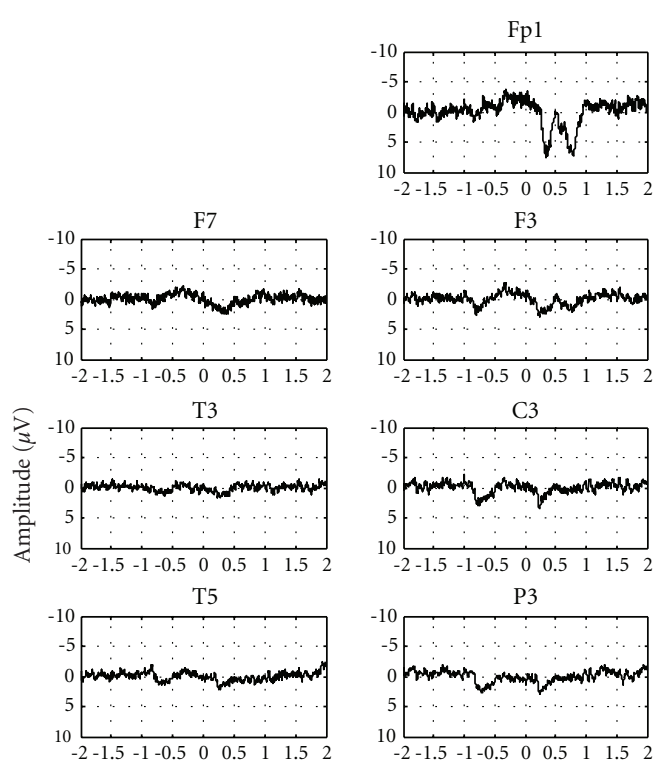

F3

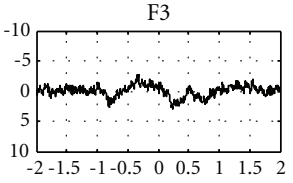

C 3

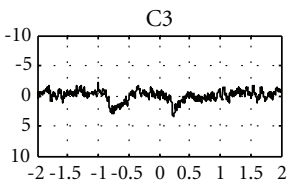

P3

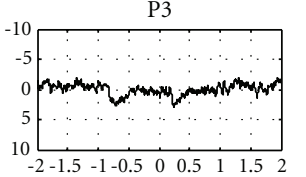

$\mathrm{O} 1$
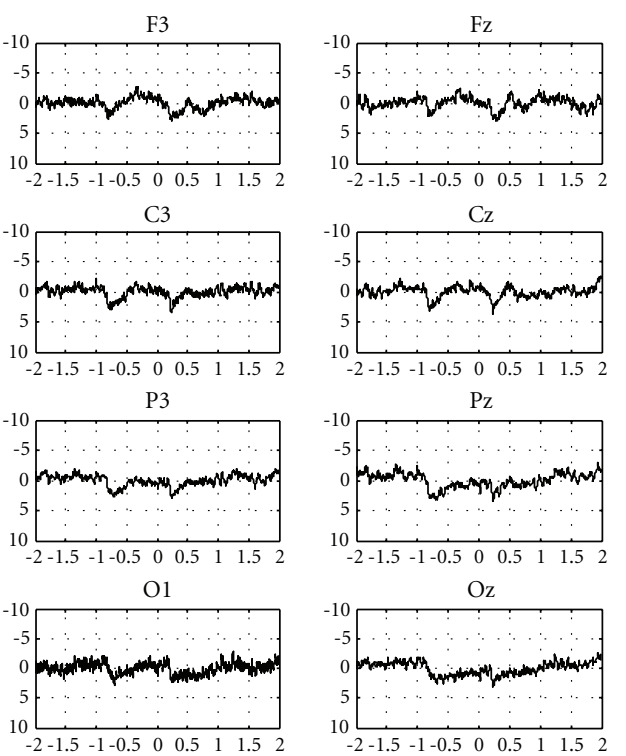

$\mathrm{Cz}$

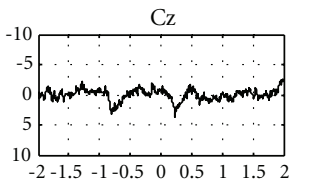

$\mathrm{Pz}$

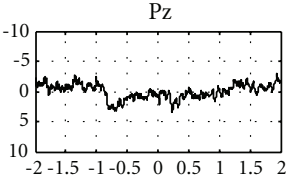

$\mathrm{Oz}$
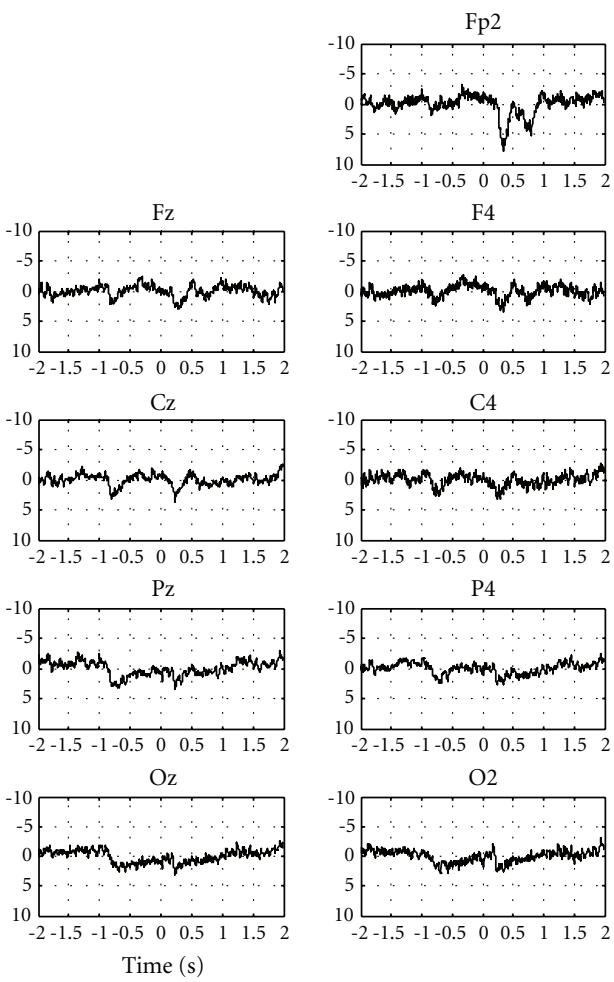

F4
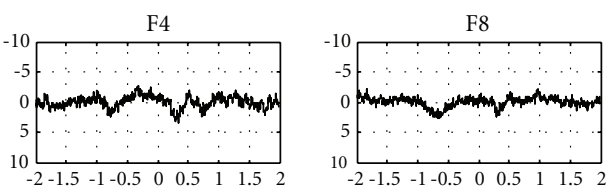

C4

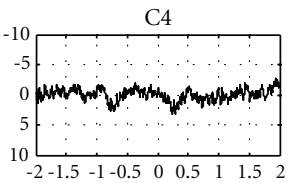

$\mathrm{T} 4$

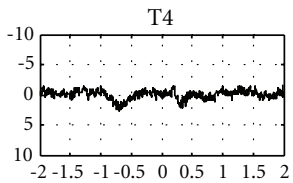

P4

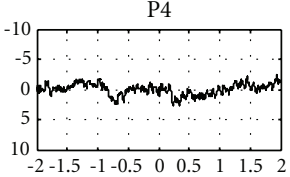

T6

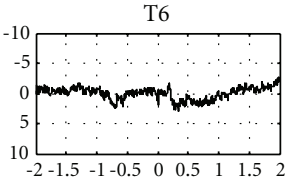

$\mathrm{O} 2$

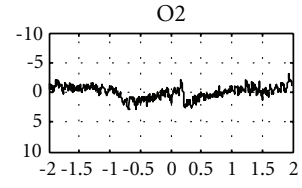

FIgURE 3: Grand Average topographic distribution of ERPs from spontaneous EEG, with lighted LEDs. 


\section{Results}

Those epochs with artifacts within the threshold (below $3 \sigma$ ) were kept in the sample. All epochs containing "strong artifacts" due to eye blinks were rejected. All ten volunteers demonstrated eye blinking artifact. Some of these artifacts occurred at the same time. This may be a tendency, as shown in literature [37].

The grand average (mean of the ERP of all subjects) resulting from spontaneous EEG without LED stimuli (Figure 2), as expected, did not present significant components in its morphology, except for Fp1 and Fp2 (due to eye blinking). The grand average for EEG when LEDs were turned on (Figure 3 ) showed a negative component between $t=-0.5$ second and $t=0$ second, with maximum amplitude around $-3 \mu \mathrm{V}$ at the frontal channels (especially $\mathrm{F} 3, \mathrm{Fz}$, and F4). Besides that, positive components due to LEDs flashing-between $t=-1$ second and $t=-0.5$ second due to yellow LED lightening and between $t=0$ second and $t=0.5$ second due to LEDs being turned off-with amplitude around $3 \mu \mathrm{V}$ can be perceived especially at frontal $(\mathrm{F} 3, \mathrm{Fz}$, and $\mathrm{F} 4)$ and central $(\mathrm{C} 3, \mathrm{Cz}$, and $\mathrm{C} 4)$ areas.

The ERPs due to movement grand average (Figure 4) displays movement related potential, known as Bereitschaftspotential (BP) [38-41], symmetrically distributed in both hemispheres especially at frontal (F3, Fz, and F4), central $(\mathrm{C} 3, \mathrm{C} 4$, and $\mathrm{Cz})$ and parietal $(\mathrm{P} 3, \mathrm{Pz}$, and $\mathrm{P} 4)$ channels. These BPs begin around 0.5 second before actual movement and end with the movement's outset. With the movement onset ( $t=0.25$ second) the potential declines, followed by a positive component at $t=0.7$ second, lasting approximately 1 second. Some responses at the T3 and T4 electrodes, although with smaller amplitude, are also identified. Among those identified regions, the central (motor) region displays ERPs with the largest amplitude, with $\mathrm{Cz}$ and $\mathrm{C} 4$ (contralateral movement) showing the largest amplitude.

Movement imagination ERPs (grand average) topographic disposition (Figure 5) shows that these ERPs are more consistent in the frontal, central, and parietal regions. These potentials show a negative component that begin at $t=-0.5$ second lasting approximately 1 second. After the command to the imagination ( $t=0$ second) the potential suffers a decline that can remain about 1 second. Responses with smaller amplitudes were also observable at the temporal $\mathrm{T} 3$ and $\mathrm{T} 4$ electrodes.

For the statistic detection of event response, by means of MSC, EEG epochs were used, which began 0.7 second before the order for the movement, actual or imaginary, $(t=0$ second) up to 2 seconds after the order. This interval was chosen, so that the evoked potential due to the visual stimulus (lighting of the red LED in $t=-4$ second and of the yellow LED in $t=-1$ second) does not interfere with the results-the evoked potential goes up to 125 milliseconds after the stimulus [41]. The application of MSC (with $M=45$ ), in the channel $\mathrm{Cz}$, for subject \#10's, during the spontaneous EEG, with the LEDs both unlighted (Figure 6(a)) and lighted (Figure 6(b)), shows an absence of response, as was expected. Few harmonics (4.8\% of total samples; Figures 6(a) and 6(b)) exceeded the value of $\mathrm{MSC}_{\text {critic }}=0.0658$, being within the false positives for $\alpha=5 \%$. Similar results were obtained for all volunteers, as shown in the histograms of Figures 8 and 9.

During movement, MSC identified a consistent response at $0.3-2 \mathrm{~Hz}$ band (Figure $7(\mathrm{a})$ ). The histogram in Figure 10 shows that the majority of subjects demonstrated detection in this band in the frontal, central, and parietal regions. All subjects showed some response at C4 (contralateral). There was also some detection at temporal and occipital regions.

For movement imagination, MSC detected response at $0.3-1 \mathrm{~Hz}$ band (Figure 7(b)). Most subjects showed response at the central region-nine out of ten at C4 (Figure 11). The Wilcoxon test for paired data, between the MSC of movement imagination and spontaneous EEG (unlighted LEDs), corroborated the statistical difference with a significance level of $P<.02$ for the band of 0.1 to $0.8 \mathrm{~Hz}$. No other frequency band, up to $100 \mathrm{~Hz}$, presented this significant difference.

\section{Discussion}

ERP estimation results by event-synchronized epochs averaging agree, for the most part, with current literature [38-41]. Some differences are due, mostly, to the use of monopolar channels, with reference to auricular electrodes (A1 and A2), while a large proportion of current literature uses bipolar channels, with reference to Fpz electrode.

ERP estimation for spontaneous EEG with lighted LEDs presented an almost 1-second lasting potential, especially in frontal and central channels after the yellow LED was turned on (movement preparation). According to Grey Walter et al. [42], repetitive sessions of movement suggested by a particular stimulus lead to generation of a preparation potential even when the movement is not actually executed. Considering that spontaneous EEG sessions happened after movement sessions, this potential can be related to movement preparation.

Bereitschaftspotentials (BPs) identified in EEG during movement seem similar to the ones identified during movement imagination at all channels being symmetrically distributed in both hemispheres, as predicted by Kalcher and Pfurtscheller [41].

MSC application showed consistent low-frequency harmonics (inferior to $4 \mathrm{~Hz}$ - delta band) detection, both for movement and movement imagination, corroborating recent results reported by Vuckovic and Sepulveda [28, 43], whose also related this band with both movement and movement imagination, using evidence from energy variation (synchronization and desynchronization-ERD/ERS), in bipolar channels in central, frontal, and parietal regions.

MSC technique was able to consistently detect movement imagination related response, using 45 epochs $(M=45)$, with signal-noise ration (SNR) appropriate to delta band detection in the subject group. However, 3 subjects had to go over data collection sessions up to three times, before responses could be reliably detected in their data. This variation can be explained by the fact that response SNR is closely linked to imagination abilities of each individual subject [44]. For instance, subject \#1's maximum MSC 

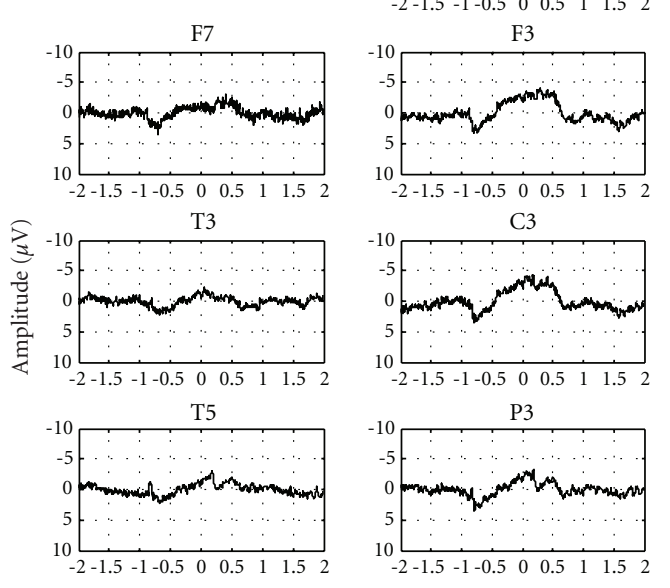
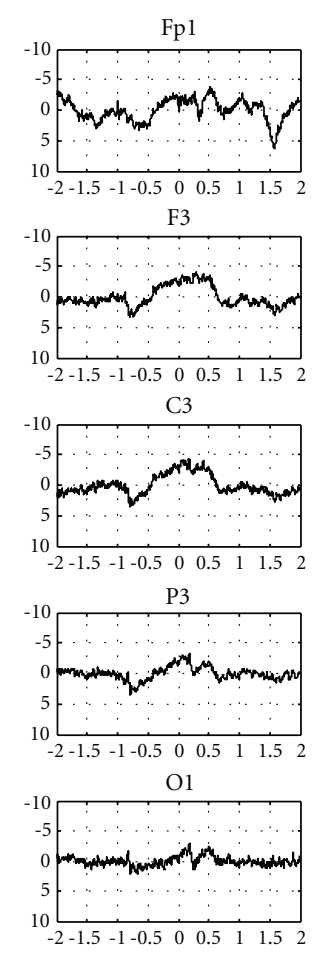

$\mathrm{C} 3$

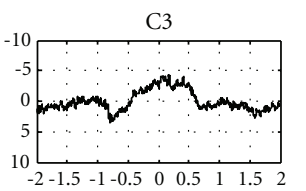

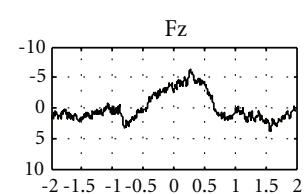
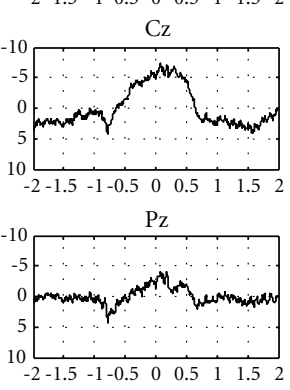

$\mathrm{Oz}$

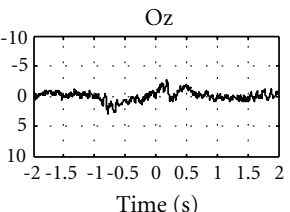

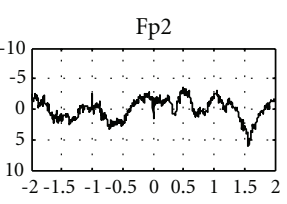

$\mathrm{F} 4$
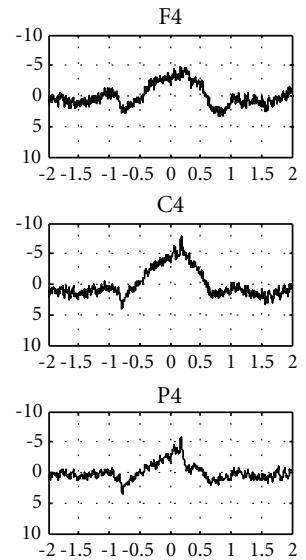

$\mathrm{O} 2$

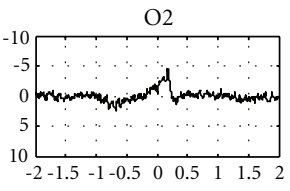

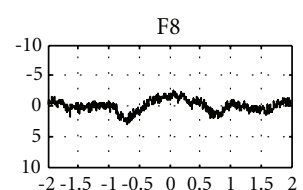

$\mathrm{T} 4$

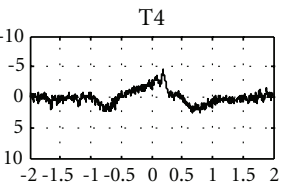

T6

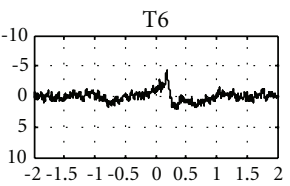

$\begin{array}{llllllll}10 & -1.5 & -1 & -0.5 & 0 & 0.5 & 1 & 1.5\end{array}$

FIGURE 4: Grand Average topographic distribution of ERPs related to left index finger movement.
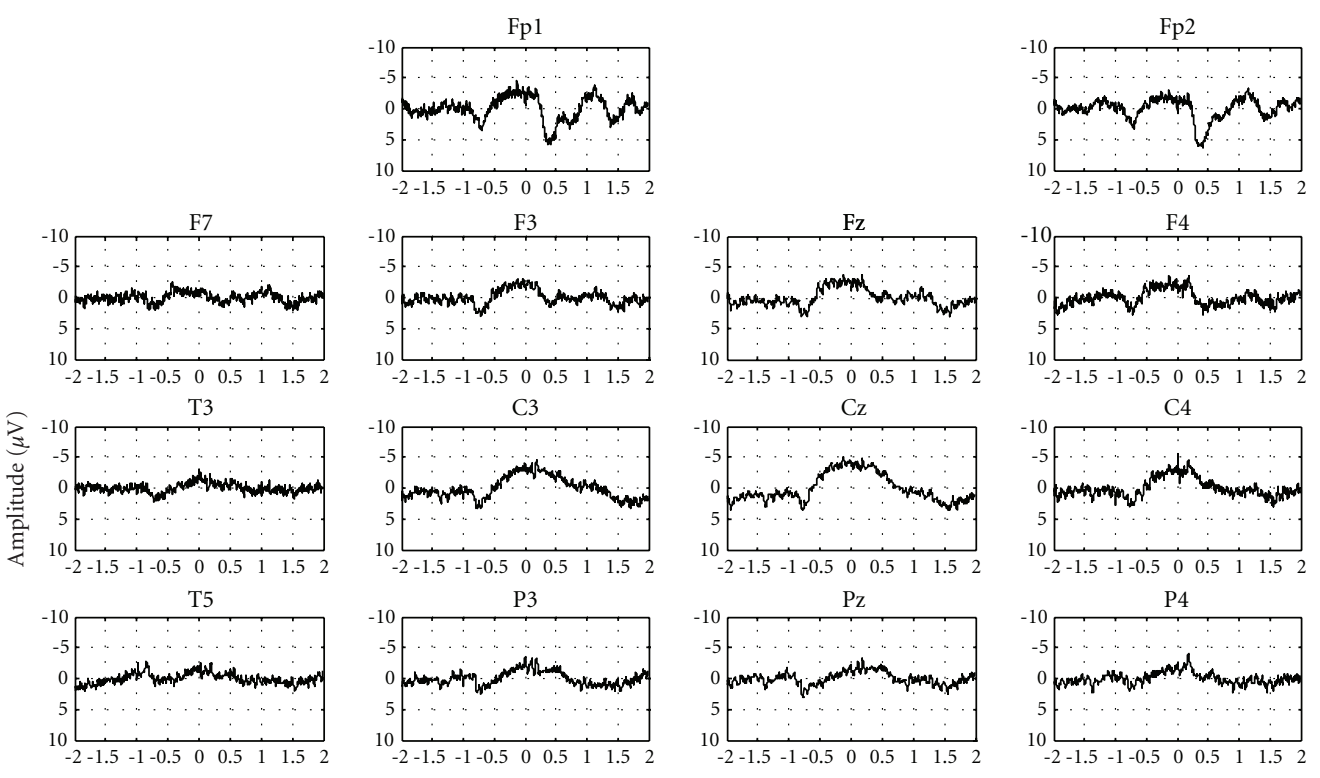

$\mathrm{F} 4$

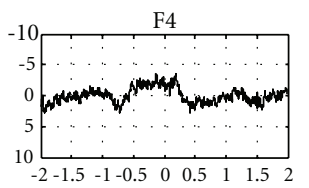

$\mathrm{C} 4$

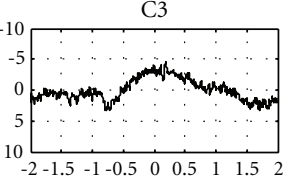

$\mathrm{Cz}$

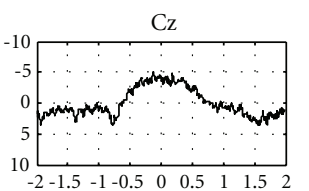

P3

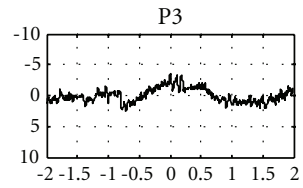

$\mathrm{O} 1$
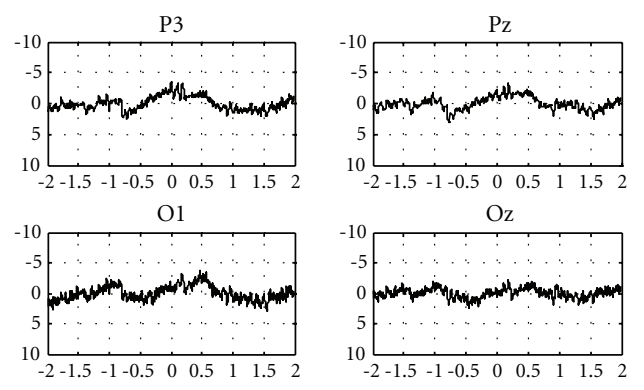

$\mathrm{Oz}$
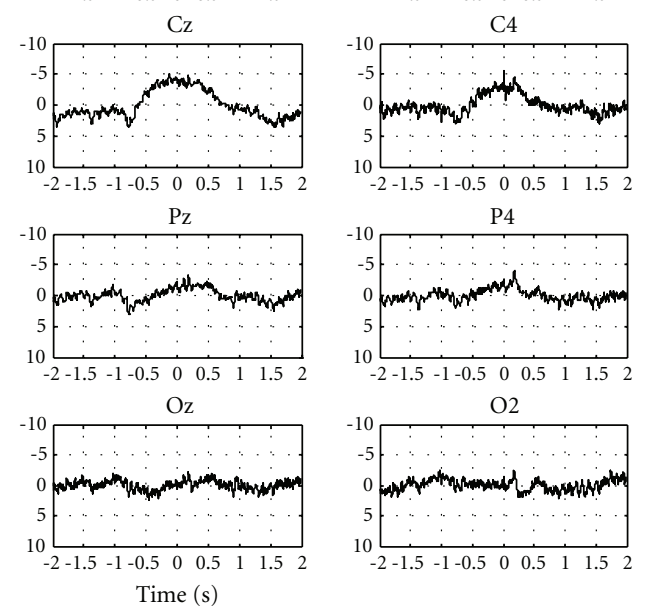

$\mathrm{P} 4$

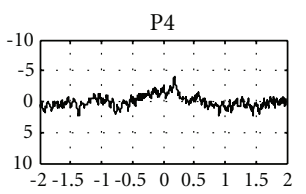

$\mathrm{O} 2$
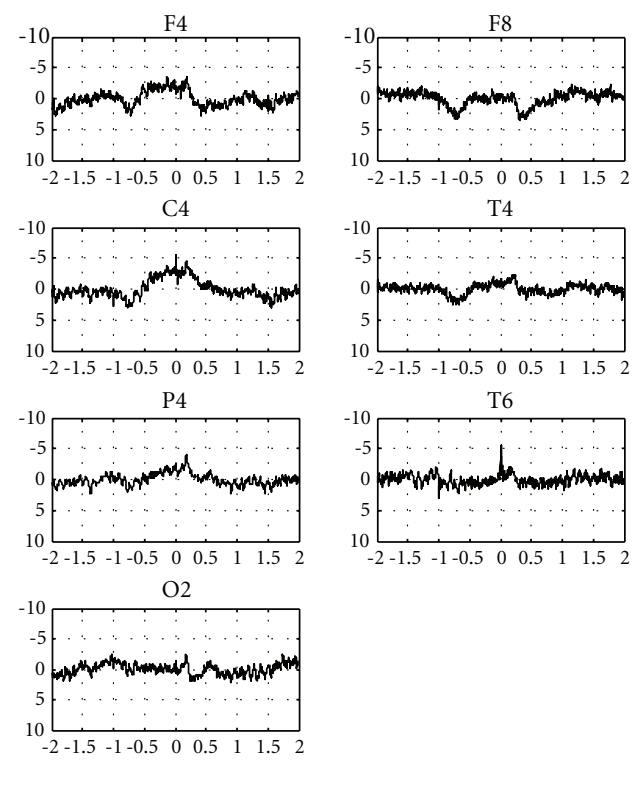

$\mathrm{T} 4$
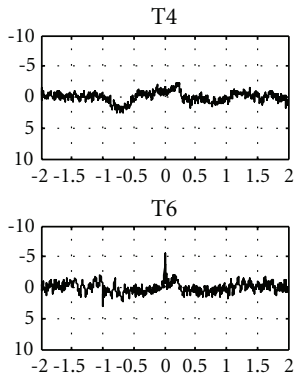

FIgURE 5: Grand Average topographic distribution of ERPs related to left index finger movement imagination. 


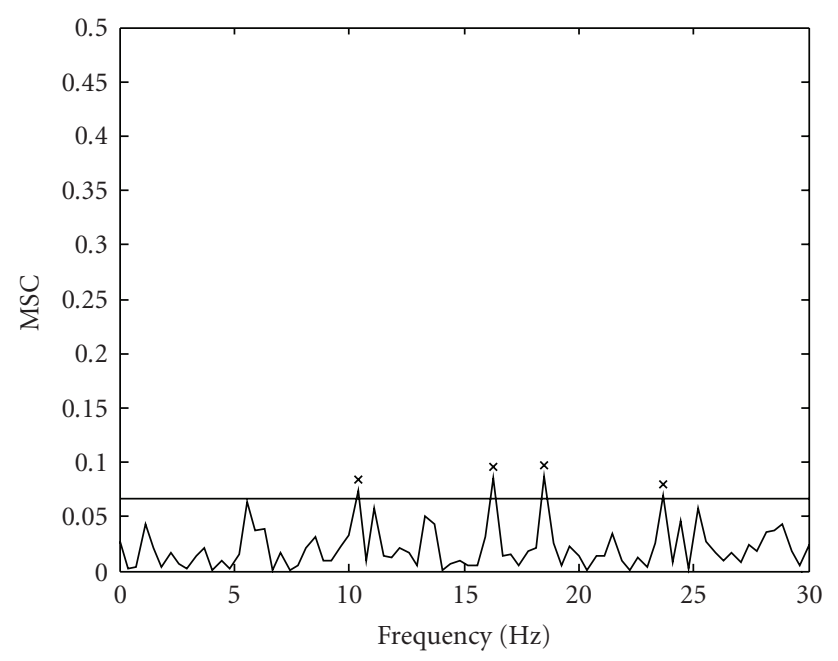

(a)

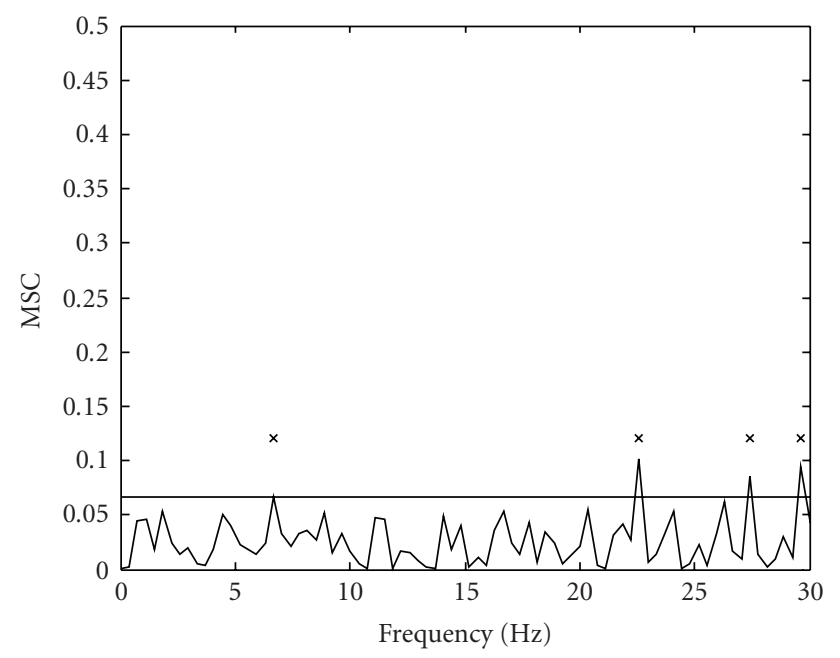

(b)

FIGURE 6: MSC $(M=45)$ for subject \#10's spontaneous EEG at $\mathrm{Cz}$ channel with (a) unlighted LEDs and (b) lighted LEDs. The critical value $\left(\mathrm{MSC}_{\text {critic }}=0.0658\right.$, for $\alpha=5 \%$ ) is represented by the horizontal line. Similar behavior found frequencies higher than $30 \mathrm{~Hz}$.

for movement imagination was 0.452 (Figure 12(a)), being superior to subject \#10's (maximum MSC $=0.248$ ), as depicted by Figure 7(b). The coherence value reached by MSC for subject \#1 makes ERP detection possible with considerably fewer epochs $(M=10$, Figure 12(b)).

ERPs related to LEDs flashing (yellow LED lightening and both LEDs turning off) did not exhibit any influence on movement imagination detection by MSC. Those ERPs presented the highest MSC values, around $3 \mathrm{~Hz}$ that are higher than those of movement imagination MSC band (around $1 \mathrm{~Hz}$ ), as shown in Figure 13. Eye blinking potentials can also influence response detection, particularly at Fp1 and Fp2 channels. Even with artifact rejection algorithm application and event-synchronized epochs averaging technique, some synchronous activity during eye blinking was still observable in the moment subjects were instructed to initiate

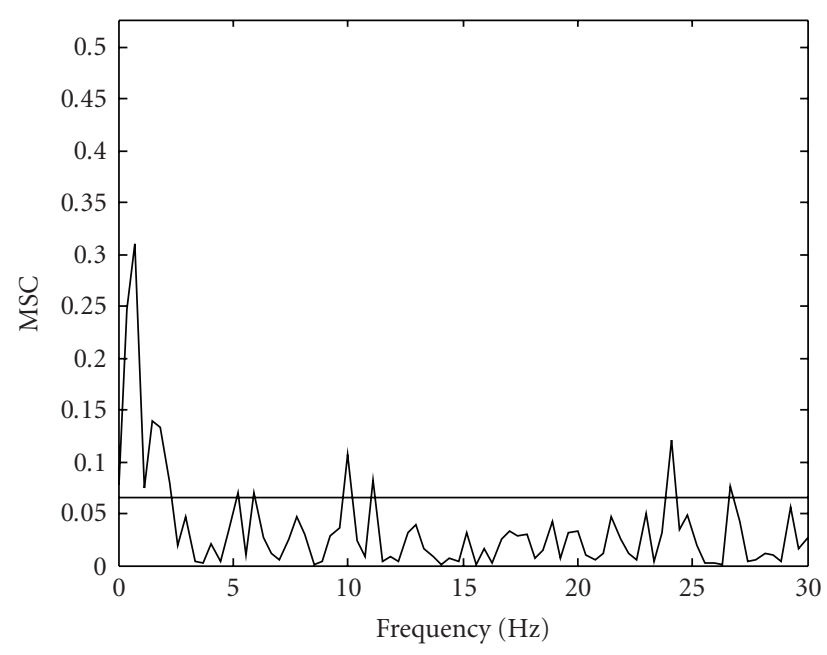

(a)

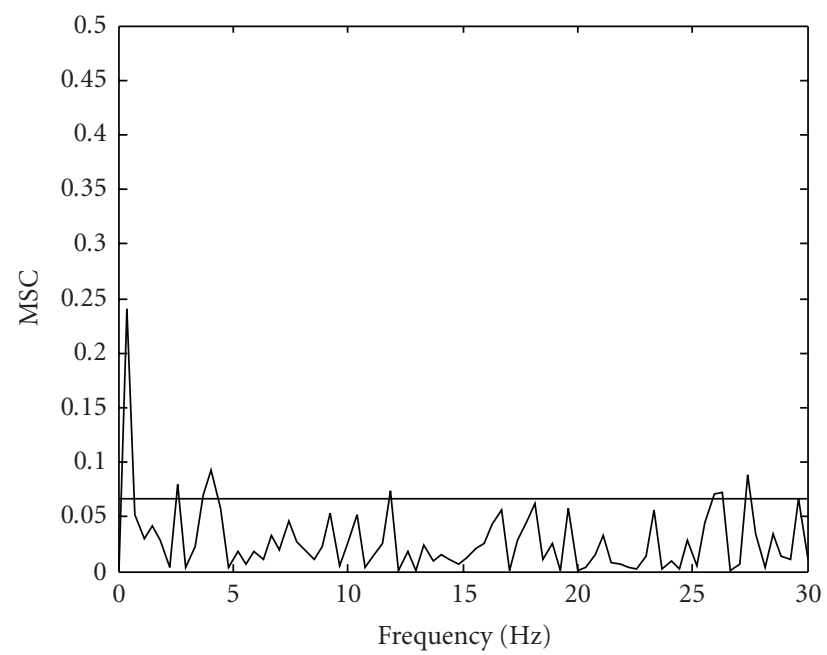

(b)

Figure 7: MSC $(M=45)$ for subject \#10's at Cz channel. (a) During left index finger movement. (b) During left index finger movement imagination. The critical value $\left(\mathrm{MSC}_{\text {critic }}=0.0658\right)$ is represented by the horizontal line. For frequencies higher than $30 \mathrm{~Hz}$ there was no response detection.

the task (LEDs turned off) in different sessions (except during unlighted LEDs session). Recent studies have shown that eye blinking bears a clear relation to cognitive tasks [37]. Nonetheless, when it comes to BCI applications related to movement imagination, those channels are normally not taken into consideration.

Considering brain plasticity when exposed to repetitive action [45] is plausible to expect that if subjects engage in training sessions (to practice movement imagination), it is possible to improve signal-noise ratio (SNR) and therefore to reduce the amount of epochs necessary for ERP detection using MSC.

Another possible way to increment the probability detection of ERP is the application of Multiple Squared Magnitude Coherence (MSMC) proposed by $[46,47]$. This technique uses signals from more than one channel (electrode) to 

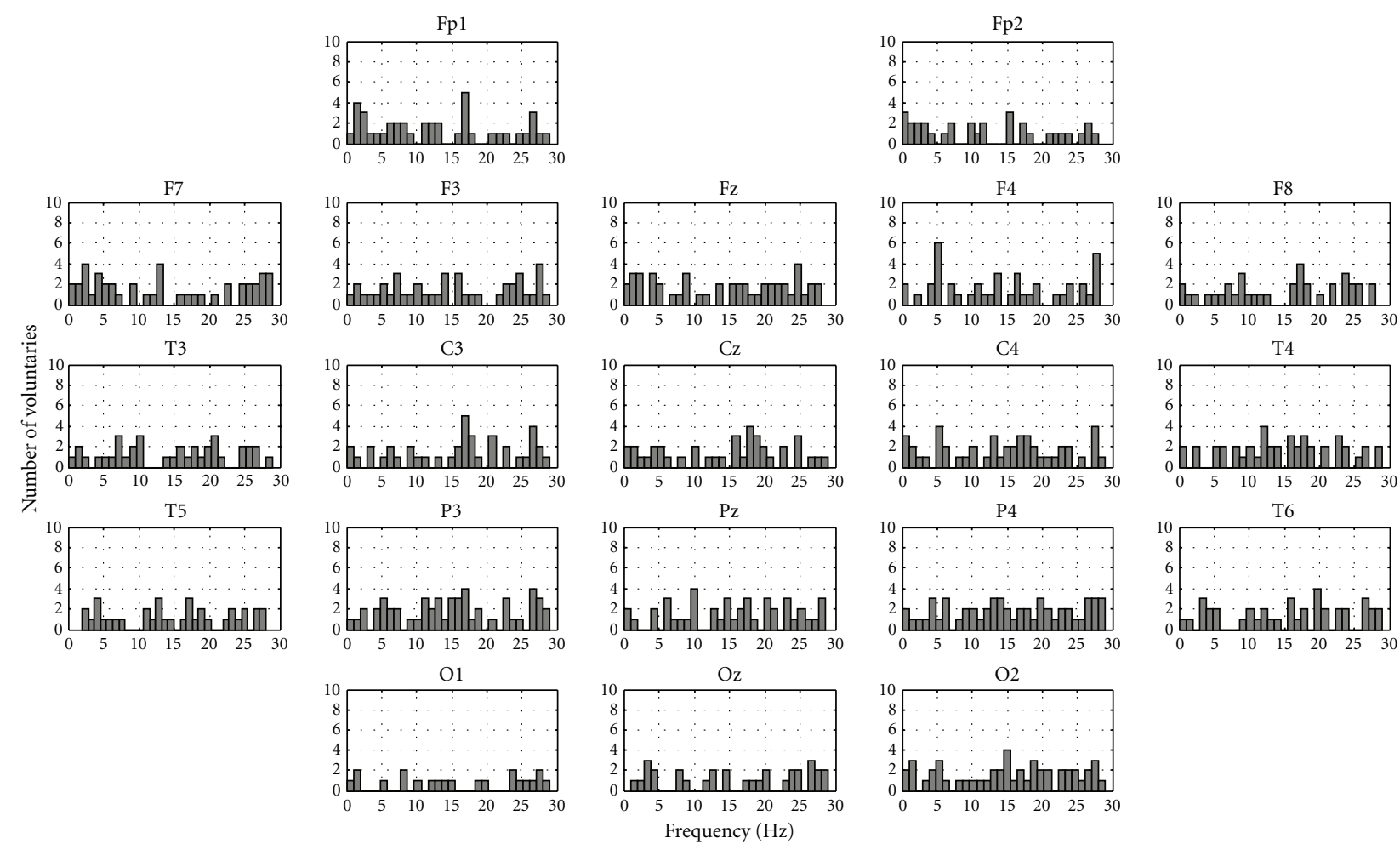

FIGURE 8: Histogram (bin width $1 \mathrm{~Hz}$ ) topographic distribution displaying the number of subjects with response after MSC application higher than the critical value (significance level 5\%) for spontaneous EEG's ERPs, with unlighted LEDs, harmonics from 0 to $30 \mathrm{~Hz}$. Frequencies higher than $30 \mathrm{~Hz}$ did not show significant responses.
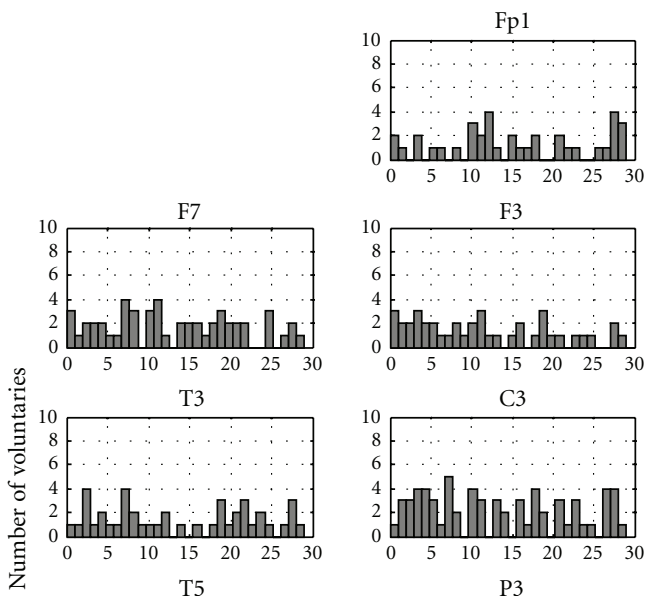

P3

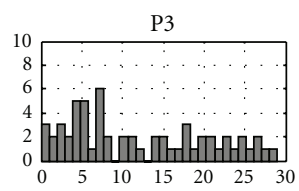

$\mathrm{O} 1$

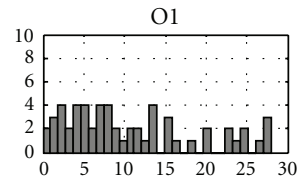

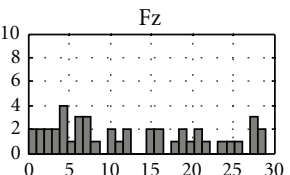

$\mathrm{Cz}$

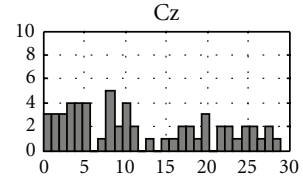

$\mathrm{Pz}$

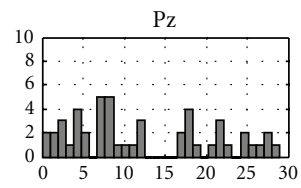

$\mathrm{Oz}$

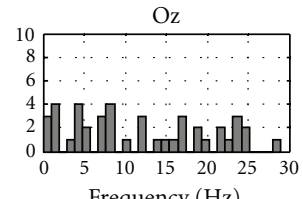

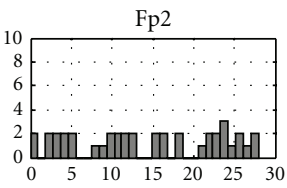
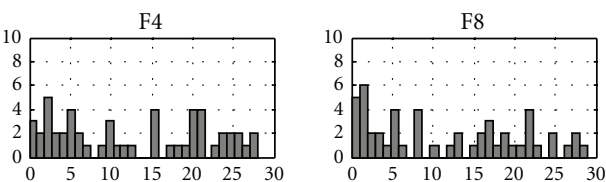

C4

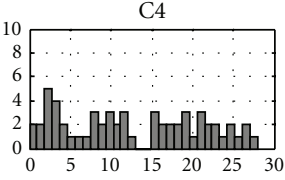

T4

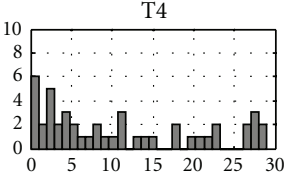

T6

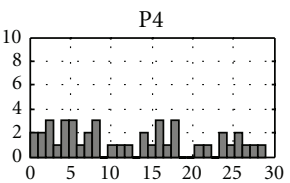

$\mathrm{O} 2$
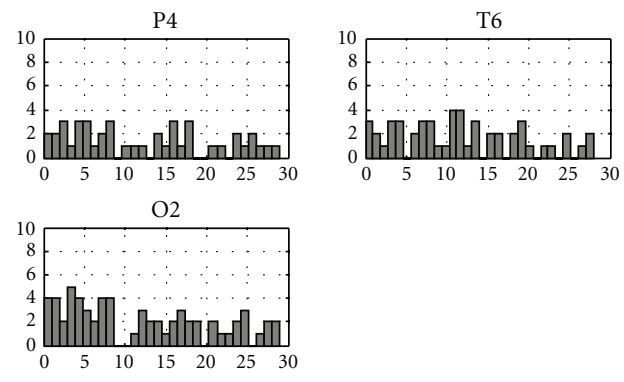

FIGURE 9: Histogram (bin width $1 \mathrm{~Hz}$ ) topographic distribution displaying the number of subjects with response after MSC application higher than the critical value (significance level 5\%) for spontaneous EEG's ERPs, with lighted LEDs, harmonics from 0 to $30 \mathrm{~Hz}$. Frequencies higher than $30 \mathrm{~Hz}$ did not show significant responses. 

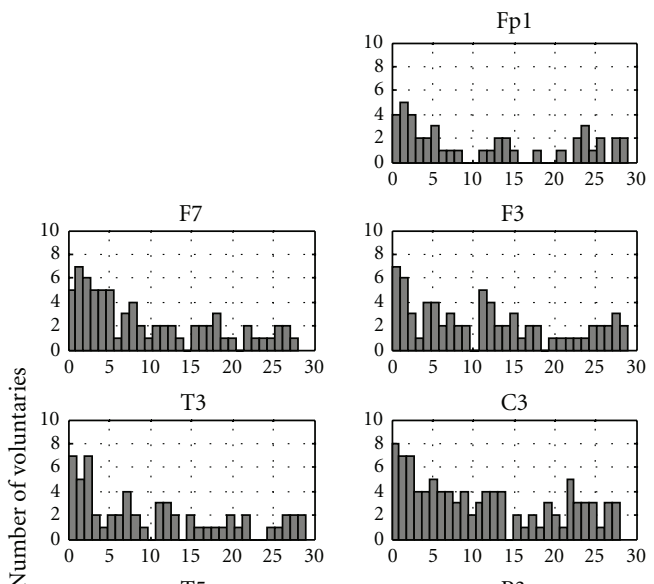

C3

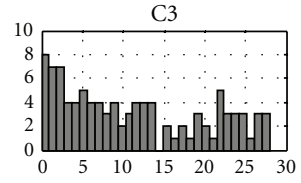

P3

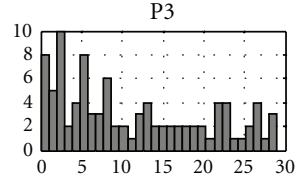

O1

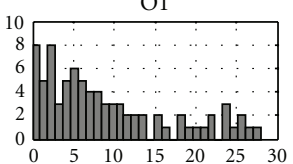

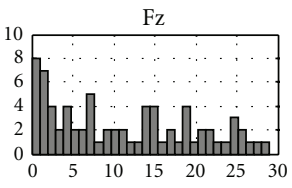

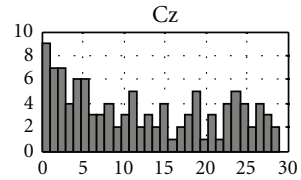

$\mathrm{Pz}$

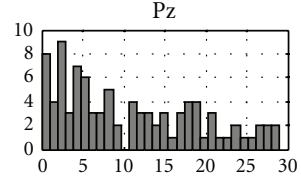

$\mathrm{Oz}$

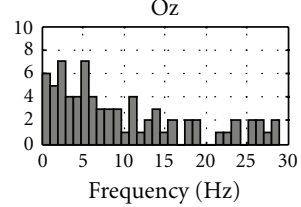

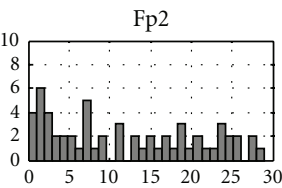

F4
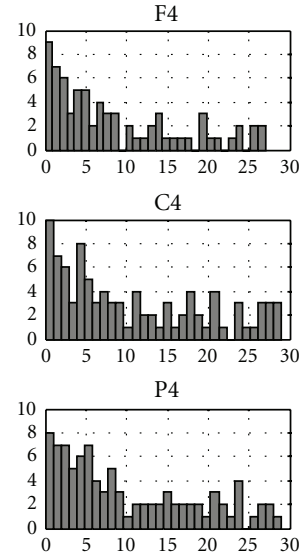

$\mathrm{O} 2$

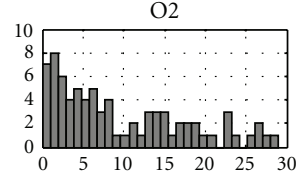

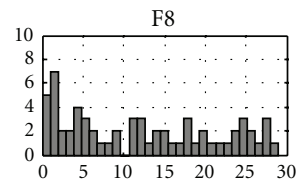

$\mathrm{T} 4$

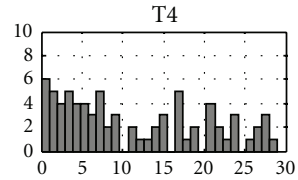

T6

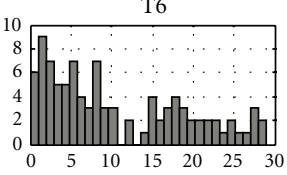

FIGURE 10: Histogram (bin width $1 \mathrm{~Hz}$ ) topographic distribution displaying the number of subjects with response after MSC application higher than the critical value (significance level 5\%) for ERPs during left index finger movement, harmonics from 0 to $30 \mathrm{~Hz}$. Frequencies higher than $30 \mathrm{~Hz}$ did not show significant responses.

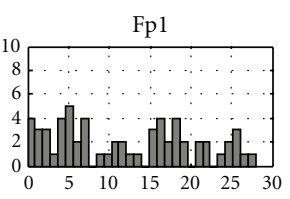

F3
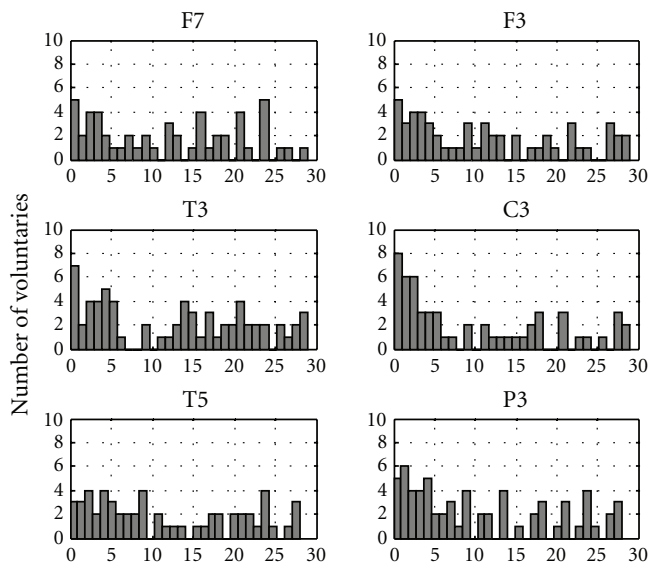

C3

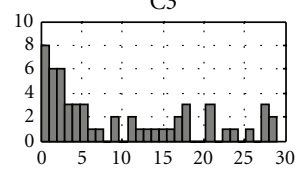

P3

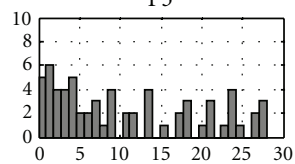

$\mathrm{O} 1$

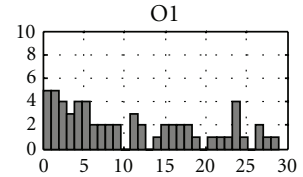

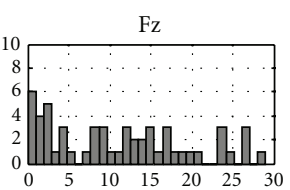

$\mathrm{Cz}$

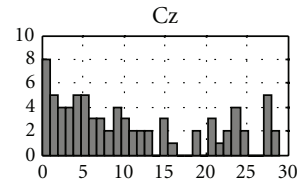

$\mathrm{Pz}$

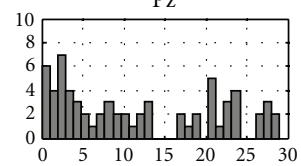

$\mathrm{Oz}$

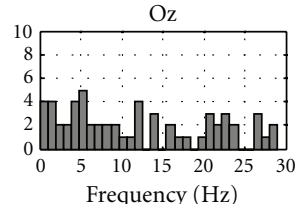

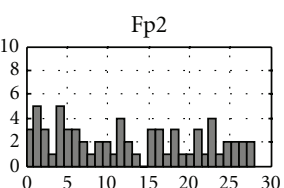

F4

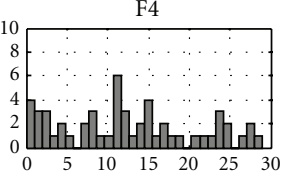

$\mathrm{C} 4$

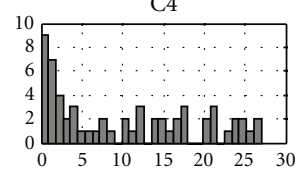

P4

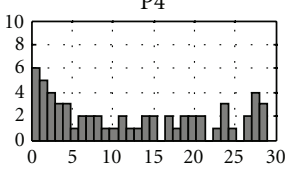

$\mathrm{O} 2$

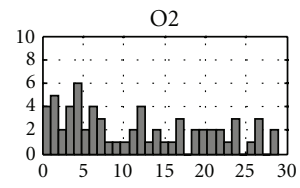

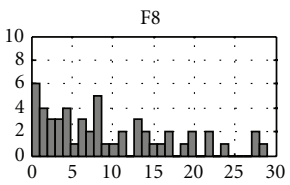

$\mathrm{T} 4$

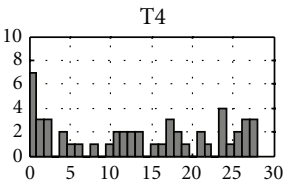

T6

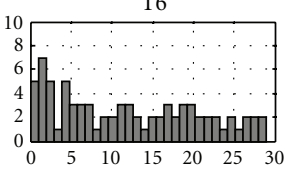

FIGURE 11: Histogram (bin width $1 \mathrm{~Hz}$ ) topographic distribution displaying the number of subjects with response after MSC application higher than the critical value (significance level 5\%) for ERPs during left index finger movement imagination, harmonics from 0 to $30 \mathrm{~Hz}$. Frequencies higher than $30 \mathrm{~Hz}$ did not show significant responses. 


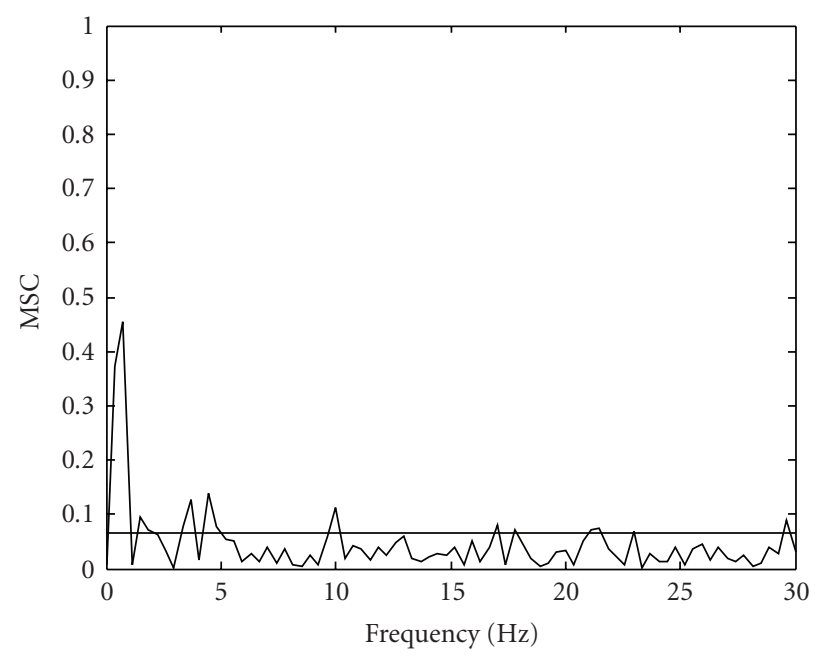

(a)

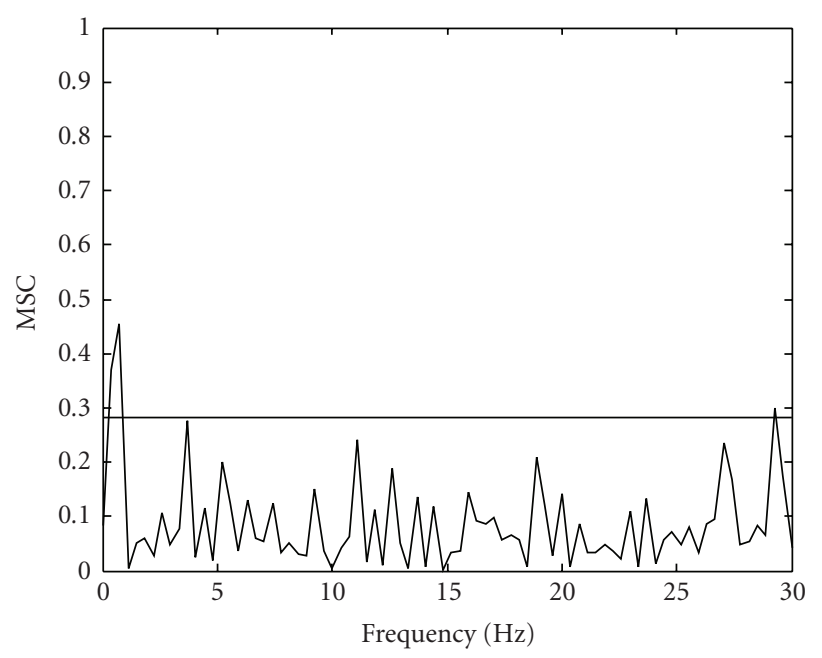

(b)

FIGURE 12: ERP statistical detection (Cz channel) related to movement imagination, for subject \#1, using the MSC (a) $M=45$; (b) $M=10$.

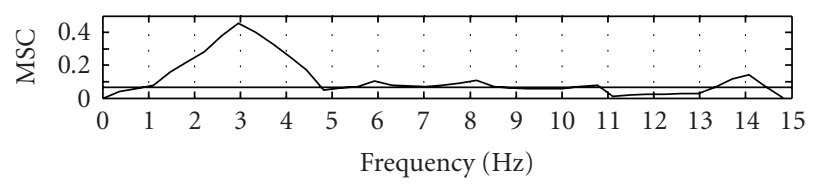

(a)

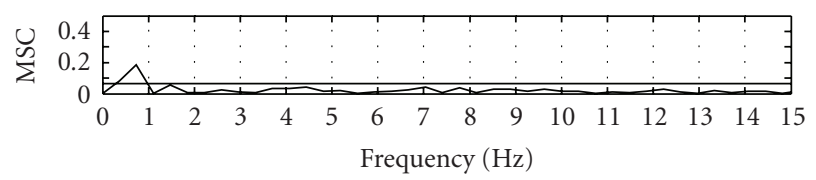

(c)

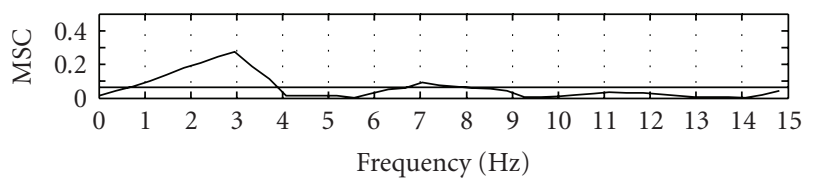

(b)

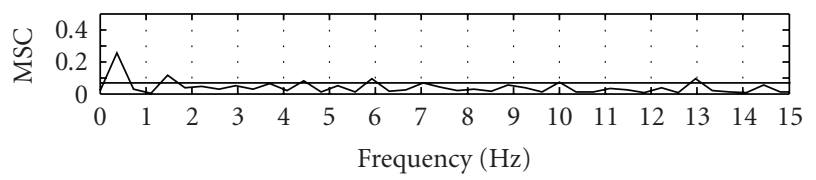

(d)

FIGURE 13: MSC for ERP due to (a) turned off LEDs, (b) yellow LED turned on, (c) actual movement, and (d) movement imagination for subject \#2. The critical value $\left(\mathrm{MSC}_{\text {critic }}=0.0658\right)$ is represented by the horizontal line.

determine brain response coherence to events. In the present investigation, the results indicate that the most suitable channels for movement imagination detection, using MSMC in 0.3 to $1 \mathrm{~Hz}$ band, are in frontal and central areas.

BCIs are usually based on single-trial methods. Thus, the MMSC with single-trial, associated with tools used for SNR improvement-techniques like Wavelet transform, principal component analyses, and independent component analysis—could be a good alternative for BCI application.

\section{Conclusion}

The present paper compared movement imagination ERPs to spontaneous EEG's ERPs (with unlighted and lighted LEDs) and to movement ERPs, demonstrating that the obtained responses were reliably related to movement imagination.

The MSC application in previously identified areas, by event-synchronized epochs averaging, proved to be an effective tool in movement imagination ERP detection. Although these detection results have been reached for 45
EEG epochs, comparable detection level was obtained with as little as 10 epochs for some subjects. We believe that with proper subject training and through multiple squared magnitude coherence alongside with noise removal tools can lead to superior detection levels. Therefore, MSC use for movement imagination ERP detection seems to be a valuable pathway for BCI development.

\section{Acknowledgments}

The authors gratefully acknowledge FAPEMIG, CNPq, and CAPES for financial support.

\section{References}

[1] P. Gnanayutham, C. Bloor, and G. Cockton, "Discrete acceleration and personalised tiling as brain-body interface paradigms for neurorehabilitation," in Proceedings of SIGCHI Conference on Human Factors in Computing Systems (CHI '05), pp. 261-270, Portland, Or, USA, April 2005. 
[2] J. M. Belsh and P. L. Schiffman, Amyotrophic Lateral Sclerosis: Diagnosis and Management for the Clinician, Future Publishing, Armonk, NY, USA, 1996.

[3] B. Kotchoubey, S. Lang, S. Winter, and N. Birbaumer, "Cognitive processing in completely paralyzed patients with amyotrophic lateral sclerosis," European Journal of Neurology, vol. 10, no. 5, pp. 551-558, 2003.

[4] J. K. Chapin, K. A. Moxon, R. S. Markowitz, and M. A. L. Nicolelis, "Real-time control of a robot arm using simultaneously recorded neurons in the motor cortex," Nature Neuroscience, vol. 2, no. 7, pp. 664-670, 1999.

[5] N. G. Hatsopoulos, J. Joshi, and J. G. O'Leary, "Decoding continuous and discrete motor behaviors using motor and premotor cortical ensembles," Journal of Neurophysiology, vol. 92, no. 2, pp. 1165-1174, 2004.

[6] M. A. L Nicolelis and S. Ribeiro, "Multielectrode recordings: the next steps," Current Opinion in Neurobiology, vol. 12, no. 5, pp. 602-606, 2002.

[7] S. P. Levine, J. E. Huggins, S. L. BeMent, et al., "A direct brain interface based on event-related potentials," IEEE Transactions on Rehabilitation Engineering, vol. 8, no. 2, pp. 180-185, 2000.

[8] P. R. Kennedy, R. A. E. Bakay, M. M. Moore, K. Adams, and J. Goldwaithe, "Direct control of a computer from the human central nervous system," IEEE Transactions on Rehabilitation Engineering, vol. 8, no. 2, pp. 198-202, 2000.

[9] P. G. Patil, J. M. Carmena, M. A. L. Nicolelis, and D. A. Turner, "Ensemble recordings of human subcortical neurons as a source of motor control signals for a brain-machine interface," Neurosurgery, vol. 55, no. 1, pp. 35-38, 2004.

[10] E. C. Leuthardt, G. Schalk, J. R. Wolpaw, J. G. Ojemann, and D. W. Moran, "A brain-computer interface using electrocorticographic signals in humans," Journal of Neural Engineering, vol. 1, no. 2, pp. 63-71, 2004.

[11] B. Graimann, J. E. Huggins, S. P. Levine, and G. Pfurtscheller, "Toward a direct brain interface based on human subdural recordings and wavelet-packet analysis," IEEE Transactions on Biomedical Engineering, vol. 51, no. 6, pp. 954-962, 2004.

[12] P. Forslund, A neural network based brain-computer interface for classification of movement related EEG, M.S. thesis, Linkoping University, Linkoping, Sweden, 2003.

[13] R. Krepki, G. Curio, B. Blankertz, and K.-R. Müller, "Berlin Brain-Computer Interface. The HCI communication channel for discovery," International Journal of Human Computer Studies, vol. 65, no. 5, pp. 460-477, 2007.

[14] T. Hinterberger, A. Kübler, J. Kaiser, N. Neumann, and N. Birbaumer, "A brain-computer interface (BCI) for the lockedin: comparison of different EEG classifications for the thought translation device," Clinical Neurophysiology, vol. 114, no. 3, pp. 416-425, 2003.

[15] J.-Z. Xue, H. Zhang, C.-X. Zheng, and X.-G. Yan, "Wavelet packet transform for feature extraction of EEG during mental tasks," in Proceedings of the 2nd International Conference on Machine Learning and Cybernetics, vol. 1, pp. 360-363, 2003.

[16] B.-H. Yang, G.-Z. Yan, R.-G. Yan, and T. Wu, "Adaptive subject-based feature extraction in brain-computer interfaces using wavelet packet best basis decomposition," Medical Engineering and Physics, vol. 29, no. 1, pp. 48-53, 2007.

[17] W. D. Penny, S. J. Roberts, E. A. Curran, and M. J. Stokes, "EEG-based communication: a pattern recognition approach," IEEE Transactions on Rehabilitation Engineering, vol. 8, no. 2, pp. 214-215, 2000.

[18] J. R. Wolpaw, D. J. McFarland, and T. M. Vaughan, "Braincomputer interface research at the Wadsworth Center," IEEE Transactions on Rehabilitation Engineering, vol. 8, no. 2, pp. 222-226, 2000.
[19] C. Vidaurre, A. Schlöogl, R. Cabeza, R. Scherer, and G. Pfurtscheller, "A fully on-line adaptive BCI," IEEE Transactions on Biomedical Engineering, vol. 53, no. 6, pp. 1214-1219, 2006.

[20] F. Piccione, F. Giorgi, P. Tonin, et al., "P300-based brain computer interface: reliability and performance in healthy and paralysed participants," Clinical Neurophysiology, vol. 117, no. 3, pp. 531-537, 2006.

[21] J. Kalcher and G. Pfurtscheller, "Discrimination between phase-locked and non-phase-locked event-related EEG activity," Electroencephalography and Clinical Neurophysiology, vol. 94, no. 5, pp. 381-384, 1995.

[22] C. Neuper and G. Pfurtscheller, "Motor imagery and ERD," in Event-Related Desynchronization. Handbook of Electroencephalography and Clinical Neurophysiology, G. Pfurtscheller and F. H. Lopes da Silva, Eds., vol. 6, pp. 303-325, Elsevier, Amsterdam, The Netherlands, 1999.

[23] G. Pfurtscheller and F. H. Lopes da Silva, "Event-related EEG/MEG synchronization and desynchronization: basic principles," Clinical Neurophysiology, vol. 110, no. 11, pp. 1842-1857, 1999.

[24] G. Pfurtscheller, "Quantification of ERD and ERS in the time domain," in Event-Related Desynchronization. Handbook of Electroencephalography and Clinical Neurophysiology, G. Pfurtscheller and F. H. Lopes da Silva, Eds., vol. 6, pp. 89-105, Elsevier, Amsterdam, The Netherlands, 1999.

[25] S. Makeig, A. Bell, J. T. P. Jung, and T. J. Sejnowski, "Independent component analysis of electroencephalographic data," in Advances in Neural Information Processing Systems 8, D. Touretzky, M. Mozer, and M. Hasselmo, Eds., pp. 145-151, MIT Press, Cambridge, Mass, USA, 1996.

[26] S. Makeig, S. Enghoff, T. P. Jung, and T. J. Sejnowski, "A natural basis for efficient brain-actuated control," IEEE Transactions on Rehabilitation Engineering, vol. 8, no. 2, pp. 208-211, 2000.

[27] E. Q. Braga and C. J. Tierra-Criollo, "SOBI with robust orthogonalization to remove the artefact stimulus in evoked potential: $5 \mathrm{~Hz}$ current sinusoidal stimulus," in Proceedings of the 1st International Conference on Bio-Inspired System and Signal Processing (BIOSIGNALS '08), vol. 1, pp. 273-276, Funchal, Portugal, 2008.

[28] A. Vuckovic and F. Sepulveda, "Delta band contribution in cue based single trial classification of real and imaginary wrist movements," Medical and Biological Engineering and Computing, vol. 46, no. 6, pp. 529-539, 2008.

[29] H. Ramoser, J. Muller-Gerking, and G. Pfurtscheller, "Optimal spatial filtering of single trial EEG during imagined hand movement," IEEE Transactions on Rehabilitation Engineering, vol. 8, no. 4, pp. 441-446, 2000.

[30] N. Birbaumer, "Brain-computer-interface research: coming of age," Clinical Neurophysiology, vol. 117, no. 3, pp. 479-483, 2006.

[31] D. M. Simpson, C. J. Tierra-Criollo, R. T. Leite, E. J. B. Zayen, and A. F. C. Infantosi, "Objective response detection in an electroencephalogram during somatosensory stimulation," Annals of Biomedical Engineering, vol. 28, no. 6, pp. 691-698, 2000.

[32] R. A. Dobie and M. J. Wilson, "Analysis of auditory evoked potentials by magnitude-squared coherence," Ear and Hearing, vol. 10, no. 1, pp. 2-13, 1989.

[33] A. M. F. L. Miranda de Sá, A. F. C. Infantosi, and D. M. Simpson, "Coherence between one random and one periodic signal for measuring the strength of responses in the electroencephalogram during sensory stimulation," Medical and Biological Engineering and Computing, vol. 40, no. 1, pp. 99 104, 2002. 
[34] A. M. F. L. Miranda de Sá and A. F. C. Infantosi, "Evaluating the entrainment of the alpha rhythm during stroboscopic flash stimulation by means of coherence analysis," Medical Engineering \& Physics, vol. 27, no. 2, pp. 167-173, 2005.

[35] G. C. Carter, "Tutorial overview of coherence and time delay estimation," in Coherence and Time Delay Estimation-An Applied Tutorial for Research, Developing, Test and Evaluation Engineers, G. C. Carter, Ed., pp. 1-27, IEEE Press, New York, NY, USA, 1993.

[36] A. M. F. L. Miranda de Sá and A. F. C. Infantosi, "Evaluating the relationship of non-phase locked activities in the electroencephalogram during intermittent stimulation: a partial coherence-based approach," Medical and Biological Engineering and Computing, vol. 45, no. 7, pp. 635-642, 2007.

[37] G. J. Siegle, N. Ichikawa, and S. Steinhauer, "Blink before and after you think: blinks occur prior to and following cognitive load indexed by pupillary responses," Psychophysiology, vol. 45, no. 5, pp. 679-687, 2008.

[38] K. H. Chiappa, Evoked Potentials in Clinical Medicine, Lippincott-Raven, Philadelphia, Pa, USA, 1997.

[39] J. D. Guieu, J. L. Bourriez, P. Derambure, L. Defebvre, and F. Cassim, "Temporal and spatial aspects of event-related desynchronization and movement-related cortical potentials," in Event-Related Desynchronization. Handbook of Electroencephalography and Clinical Neurophysiology, G. Pfurtscheller and F. H. Lopes da Silva, Eds., vol. 6, pp. 279-290, Elsevier, Amsterdam, The Netherlands, 1999.

[40] L. Deecke, B. Groezinger, and H. H. Kornhuber, "Voluntary finger movement in man: cerebral potentials and theory," Biological Cybernetics, vol. 23, no. 2, pp. 99-119, 1976.

[41] J. Kalcher and G. Pfurtscheller, "Discrimination between phase-locked and non-phase-locked event-related EEG activity," Electroencephalography and Clinical Neurophysiology, vol. 94, no. 5, pp. 381-384, 1995.

[42] W. Grey Walter, R. Cooper, V. J. Aldridge, W. C. McCallum, and A. L. Winter, "Contingent negative variation: an electric sign of sensori-motor association and expectancy in the human brain," Nature, vol. 203, no. 4943, pp. 380-384, 1964.

[43] A. Vuckovic and F. Sepulveda, "Quantification and visualisation of differences between two motor tasks based on energy density maps for brain-computer interface applications," Clinical Neurophysiology, vol. 119, no. 2, pp. 446-458, 2008.

[44] M. V. Stecklow, Imagética motora em tarefa complexa: análise na banda alfa do eletroencefalograma, M.S. thesis, COPPE/UFRJ, Rio de Janeiro, Brazil, 2006.

[45] A. Pascual-Leone, A. Amedi, F. Fregni, and L. B. Merabet, "The plastic human brain cortex," Annual Review of Neuroscience, vol. 28, pp. 377-401, 2005.

[46] A. M. F. L. Miranda de Sá and L. B. Felix, "Improving the detection of evoked responses to periodic stimulation by using multiple coherence-application to eeg during photic stimulation," Medical Engineering and Physics, vol. 24, no. 4, pp. 245-252, 2002.

[47] A. M. F. L. Miranda de Sá, A. F. C. Infantosi, and D. B. Melges, "A multiple coherence-based detector for evoked responses in the eeg during sensory stimulation," in Proceedings of the 30th Annual International Conference of the IEEE Engineering in Medicine and Biology Society (EMBS '08), pp. 3516-3519, 2008. 